\title{
Short communication: Effect of mutation age on genomic predictions
}

\author{
J. Casellas ${ }^{1}$ and L. Varona† \\ *Grup de Recerca en Remugants, Departament de Ciència Animal i dels Aliments, Universitat Autònoma de Barcelona, 08193 Bellaterra, Spain \\ †Departamento de Anatomía, Embriología y Genética Animal, Universidad de Zaragoza, 50013 Zaragoza, Spain
}

\section{ABSTRACT}

Genomic selection relies on the whole-genome evaluation of single nucleotide polymorphisms (SNP), some of them linked to quantitative trait loci (QTL). Although statistical methodology has been developed for the analysis of genomic data, little is known about the performance of SNP association studies when trying to capture variability from QTL mutations of different ages. Within this context, the influence of mutation age was analyzed under a simulation design, assuming presence or absence of selection on mutant QTL alleles. Focusing on a unique chromosome with a single QTL located in the proximal end, the performance of the genomic selection analyses was evaluated in terms of standardized mean square error (MSE). For all simulation scenarios, MSE was highest for the youngest mutations. The MSE was progressively reduced with mutation age under random mating and soft selection, and reached its maximum performance with the oldest mutations. On the other hand, moderate and strong selection caused a quick reduction of the MSE from youngest mutations to mutations arising in generations 920 to 939, thus resulting in a progressive increase for older mutations. In both cases, very young mutations escaped from genomic selection analyses, releasing a relevant amount of genetic variability that could not be captured and used in genomic selection programs. This demonstrated the need for new analytical approaches to model relevant and recent sources of variation; if captured, these young mutations could substantially contribute to current breeding schemes.

Key words: genomic selection, mean square error, mutation age, simulation

\section{Short Communication}

Whole-genome evaluation attempts to exploit linkage disequilibrium between SNP and QTL for genomic selection purposes. It relies mainly on the fact that additive genetic variability originated by several dozen

Received January 18, 2011.

Accepted March 31, 2011.

${ }^{1}$ Corresponding author: joaquim.casellas@uab.cat or a few hundred QTL spread in the whole genome (Hayes and Goddard, 2001) would be captured by a dense panel of SNP markers and appropriate statistical methodologies. This scenario was introduced by Meuwissen et al. (2001) and has initiated a revolution in the animal breeding field. Nevertheless, our knowledge about the relationship between the statistical performance of genomic selection and the genetic history of relevant polymorphisms remains limited.

The continuous uploading of new mutations in mammalian genomes (Casellas and Medrano, 2008; Casellas et al., 2010) could be a confounding factor for genomic selection analyses. Mixed linear models (Meuwissen et al., 2001) must deal with QTL mutations arising over a long temporal space (i.e., generations) that are at variable linkage disequilibrium with neighboring SNP (Innan and Nordborg, 2003). Although new and old mutations can be accounted for in BLUP equations (Henderson, 1973) by the numerator relationship matrix (Henderson, 1976; Quaas, 1976) and the mutational relationship matrix (Wray, 1990), performance of genomic prediction models on the basis of the age of new mutations is not well established. The study of the ability of genomic prediction models to capture genetic variability from mutations originating at different times was the main objective of this short communication.

This study was based on stochastic simulation and assumed 4 different scenarios (i.e., absence of selection on mutant alleles and 3 different selection intensities on mutant alleles). In total, 10,000 simulated populations were used to characterize the performance of genomic prediction under each scenario. Simulated populations were founded and evolved during 1,000 nonoverlapping generations with an effective population size $\left(N_{e}\right)$ of 100. This number was increased to 5,000 individuals in generation 1,001. For each individual, the genome consisted of a 50-cM chromosome with 1,001 SNP (1 SNP each $0.05 \mathrm{cM}$ ) and a unique QTL located at one end of the chromosome. Similar SNP density had been used in previous simulation studies focusing on genomic prediction procedures (Habier et al., 2009). Furthermore, the 50-cM chromosome exceeds the previously reported extend of linkage disequilibrium in mammalian genomes (Innan and Nordborg, 2003). The proximal location of the QTL was assumed to provide a 
clear characterization of linkage disequilibrium between SNP and the QTL, avoiding confusing scenarios with different linkage at each side of the QTL. Founder individuals (generation 1) had homozygous genotypes in all SNP (allele $S$ ) and the QTL location (allele $Q$ ). A mutation rate of $2.5 \times 10^{-3}$ per SNP was applied in the subsequent generations (Meuwissen et al., 2001), where mutations switched the allele state from $S$ to $s$ or vice versa. New alleles for the QTL originated at a mutation rate of $5 \times 10^{-4}$ per zygote between generations 1 and 990, whereas new mutations were prevented during the last 11 generations. This simulation pattern for the QTL was derived from the results obtained in preliminary trials in which an optimum simulation design was sought to maximize the number of replicates available for the study.

To elucidate the statistical performance of genomic prediction on the basis of the age of new mutations, only those simulated populations with a biallelic QTL maintaining the founder allele in generation 1,001 were used for further analyses. Linkage disequilibrium between adjacent loci was generated based on Kosambi's mapping function (Kosambi, 1944). The generation in which the nonfounder allele (i.e., the mutant allele) arose by mutation was recorded. The founder QTL allele $(Q)$ was assumed with null effect on the phenotype, whereas mutant alleles $(q)$ were assumed with an additive allelic effect of +0.5 . A unique phenotypic record was simulated for each individual born in generation 1,001 as the sum of the additive allelic effects from the QTL plus a residual term sampled from a standard normal distribution with mean 0 and variance 1 .

Analyses focused on 4 main simulation scenarios, each with 10,000 replicates. The first scenario assumed random mating and no selection on QTL ( NSel), whereas the other scenarios assumed direct selection on the QTL starting in generations $500\left(\boldsymbol{S e l}_{500}\right), 700$ $\left(\boldsymbol{S e l}_{\mathbf{7 0 0}}\right)$, and $900\left(\boldsymbol{S e l}_{\mathbf{9 0 0}}\right)$. Selection favored allele $q$ in all 3 latter scenarios, although selection intensity was higher in $S e l_{900}$, intermediate in $S_{e} l_{700}$, and lower in $S_{e l} l_{500}$. Selection intensity was characterized as the probability of a given individual to successfully mate and contribute offspring to the next generation. These probabilities were defined based on the QTL genotype and were specific to scenarios $S e l_{900}$ (genotype $q q=1$; $q Q$ or $Q q=0.95 ; Q Q=0.9), S_{e} l_{700}(q q=1 ; q Q$ or $Q q=$ $0.97 ; Q Q=0.94), \operatorname{Sel}_{500}(q q=1 ; q Q$ or $Q q=0.99 ; Q Q=$ $0.98)$, and $N \operatorname{Sel}(q q=1 ; q Q$ or $Q q=1 ; Q Q=1)$. Probabilities were arbitrarily chosen as a reasonable compromise between the biological plausibility of decreasing selection intensity and the probability to definitively fix (or remove) a QTL allele. Note that only those populations with a biallelic QTL containing the founder allele in generation 1,001 were retained for further studies. Given that 10,000 populations were required for each simulation scenario and the rejection rate could not be anticipated, simulations were performed back to back until 10,000 valid populations were available. At the end of the simulation process, the rejection rates were $73.3,82.8,88.7$, and $76.8 \%$ under scenarios NSel, Sel $_{500}$, $\mathrm{Sel}_{700}$, and $\mathrm{Sel}_{900}$, respectively.

Each data set was analyzed within a Bayesian context by applying the hierarchical mixed linear model reported by Gianola et al. (2003). The Bayesian likelihood was defined as multivariate normal (MVN); that is, $p\left(\mathbf{y} \mid \boldsymbol{\mu}, \boldsymbol{\gamma}, \sigma_{e}^{2}\right) \sim M V N\left(\boldsymbol{\mu}+\mathbf{M} \boldsymbol{\gamma}, \mathbf{I}_{\mathrm{e}} \sigma_{e}^{2}\right)$, where $\mathbf{y}$ was the $5,000 \times 1$ vector of phenotypic records, $\boldsymbol{\mu}$ was the 5,000 $\times 1$ vector of population means, $\mathbf{M}$ was the $5,000 \times$ 1,001 incidence matrix of known SNP genotypes, $\gamma$ was the $1,001 \times 1$ vector of random SNP effects, $\mathbf{I}_{e}$ was an identity matrix with dimensions $5,000 \times 5,000$, and $\sigma_{e}^{2}$ was the residual variance. The a priori distribution for $\gamma$ was assumed multivariate normal; that is, $p\left(\gamma \mid \sigma_{\gamma}^{2}\right) \sim \operatorname{MVN}\left(\mathbf{0}, \mathbf{I}_{\gamma} \sigma_{\gamma}^{2}\right), \mathbf{0}$ being a $1,001 \times 1$ vector of zeros, $\mathbf{I}_{\gamma}$ being an identity matrix with dimensions 1,001 $\times 1,001$, and $\sigma_{\gamma}^{2}$ being the variance of SNP effects. Improper uniform a priori distributions between $-\infty$ and $\infty$ were assumed for $\boldsymbol{\mu}$, and between 0 and $\infty$ for $\sigma_{e}^{2}$ and $\sigma_{\gamma}^{2}$. A unique Monte Carlo Markov chain (MCMC) with 210,000 elements was launched for each analysis, although only 21,000 samples were saved with a lag interval of 10 iterations. The burn-in period was smaller than 100 iterations for all replicates when determined by Raftery and Lewis (1992) on $\sigma_{\gamma}^{2}$; nevertheless, the first 1,000 iterations were discarded to ensure convergence. Mixing properties were characterized by calculating the effective chain size (Kass et al., 1998) for the $\sigma_{\gamma}^{2}$ parameter, this reporting values larger than 11,000 elements in all analyses. All unknown parameters in the model were updated by Gibbs sampling (Gelfand and Smith, 1990). After analyzing each data set, the standardized mean square error (MSE) between real (i.e., simulated; $y_{i}$ ) and predicted genetic effects (i.e., QTL effects; $\left.\tilde{y}_{i}\right)$ was calculated as MSE $=\Sigma_{i}\left(y_{i}-\tilde{y}_{i}\right)^{2} / \sigma_{Q T L}$, where $\tilde{y}_{i}$ is the sum of the posterior means from the appropriate SNP contributions and $\sigma_{Q T L}$ is the standard deviation of the QTL calculated from its allelic frequencies and the simulated additive substitution effect of its mutant alleles (i.e., q). The number of polymorphic SNP was registered and their linkage disequilibrium (LD) with the biallelic QTL was calculated as the squared correlation of the alleles at both loci $\left(\mathrm{r}^{2}\right.$; Hill and Robertson, 1968). Focusing on a given SNP, its 
Table 1. Number of replicates by simulation scenario and range of generations when the mutant QTL allele appeared

\begin{tabular}{lrrrr}
\hline & \multicolumn{4}{c}{ Simulation scenario $^{1}$} \\
\cline { 2 - 5 } Generation & NSel & Sel $_{500}$ & Sel $_{700}$ & Sel $_{900}$ \\
\hline 0 to 299 & 122 & 99 & 50 & 30 \\
300 to 499 & 355 & 197 & 113 & 82 \\
500 to 699 & 986 & 1,182 & 763 & 341 \\
700 to 799 & 975 & 1,438 & 1,238 & 477 \\
800 to 849 & 853 & 906 & 788 & 607 \\
850 to 899 & 1,158 & 1,370 & 1,402 & 1,460 \\
900 to 919 & 499 & 780 & 750 & 1,529 \\
920 to 939 & 792 & 641 & 1,150 & 1,378 \\
940 to 959 & 1,064 & 1,517 & 1,525 & 1,624 \\
960 to 979 & 1,540 & 1,202 & 1,188 & 1,180 \\
980 to 1000 & 1,656 & 10,000 & 10,000 & 10,000 \\
Overall & 10,000 & & \\
\hline
\end{tabular}

${ }^{1}$ Simulations were performed assuming no selection $(\mathrm{NSel})$ and positive selection on mutant QTL alleles with low $\left(S e l_{500}\right)$, intermediate $\left(S e l_{700}\right)$, and high $\left(S e l_{900}\right)$ selection intensities. LD with the QTL was computed as
$\mathrm{r}^{2}=\left[\left(f_{Q S}-f_{Q} f_{S}\right)^{2}\right] /\left(f_{Q} f_{q} f_{S} f_{s}\right)$, where $f_{Q}$ was the allelic
frequency of allele $Q$, and $f_{Q S}$ was the haplotypic frequency of haplotype $Q S$. All simulations and analyses were implemented with the Fortran90 programming language (Adams et al., 1992) and compiled with the GNU Fortran package (Free Software Foundation Inc., Boston, MA). The source code is available by request to the corresponding author of this paper. Results were summarized for each generation where the mutant allele appeared. More specifically, allelic frequency of the mutant QTL allele, $\mathrm{r}^{2}$, and MSE were averaged within generation interval. These intervals were defined as shown in Table 1.

Although simulations focused on a simplified mammalian genome (i.e., a unique 50-cM chromosome), this design sufficed to capture available information from SNP in LD with the QTL. Note that LD exponentially reduces with distance and becomes almost null for distances larger than $20 \mathrm{Mb}$ in cattle (Sargolzaei et al., 2008); that is, approximately $25 \mathrm{cM}(1.25 \mathrm{cM} / \mathrm{Mb}$; Arias et al., 2009). In the present study, SNP density $(\sim 2,000$ $\mathrm{SNP} / \mathrm{M}$ ) was lower than the average density provided by current commercial platforms (e.g., Bovine SNP50 BeadChip; Illumina Inc., San Diego, CA), although it agreed with recent research (Habier et al., 2009) and fell within the range of lower $(\leq 500$ markers/M; Meuwissen et al., 2001; Ødegård et al., 2009) and higher (6,000 to 10,000 SNP/M; Ibáñez-Escriche et al., 2009; Toosi et al., 2010) SNP densities reported in the scientific literature. This genetic structure may be viewed as a reasonable compromise between biological plausibility and computational complexity, providing a reliable framework where new mutations could be evaluated, based on their age, by genomic prediction methodologies.

The most relevant departures across mutation age and simulation scenarios relied on the genomic architecture (i.e., mutant QTL allele frequency and $\mathrm{r}^{2}$ ) and genomic prediction performance (i.e., MSE). It is important to note that mutation age as shown in Table 1 was categorized to obtain average allele frequency, $\mathrm{r}^{2}$, and MSE. Given that younger mutations were more abundant than older mutations (Table 1), categories for mutation age were narrower (wider) for younger (older) mutations. The change of the allelic frequency of the mutant QTL allele showed remarkably different patterns among scenarios, their maximum estimates differing at a highly significant level $(P<0.0001$; Figure 1a). Scenario $\mathrm{NSel}$ showed a progressive increase of allelic frequency with age for mutations arising during the last 3 to 5 centuries, until reaching a U-shaped distribution (results not shown) with an average estimate of $0.495 \pm 0.010$. When favorable selection was applied on the mutant QTL allele, its allelic frequency increased faster, surpassing the maximum average estimate under scenario $\mathrm{NSel}$ in fewer than 100 generations in scenarios $\mathrm{Sel}_{700}$ and $\mathrm{Sel}_{900}$ or in approximately 200 generations in scenario $S_{e l} l_{500}$. Whereas intense selection led to a pseudo-stationary pattern for allelic frequency around 0.85 in approximately 200 generations, Sel $_{500}$ maintained a positive trend, even for older mutations. These differences were expected and directly attributable to selection (Falconer and Mackay, 1996); they provide relevant information to understand further differences in average linkage disequilibrium and MSE.

Linkage disequilibrium between SNP markers and QTL becomes the basis for genomic prediction and, although partially influenced by allelic frequencies (Hill 
a)

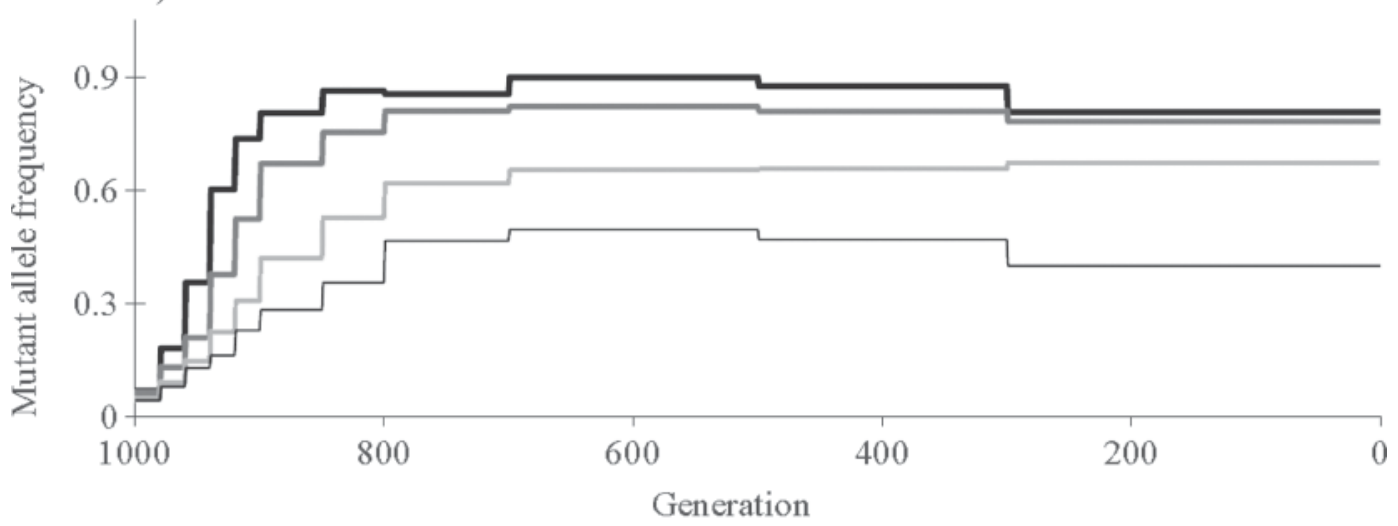

b)

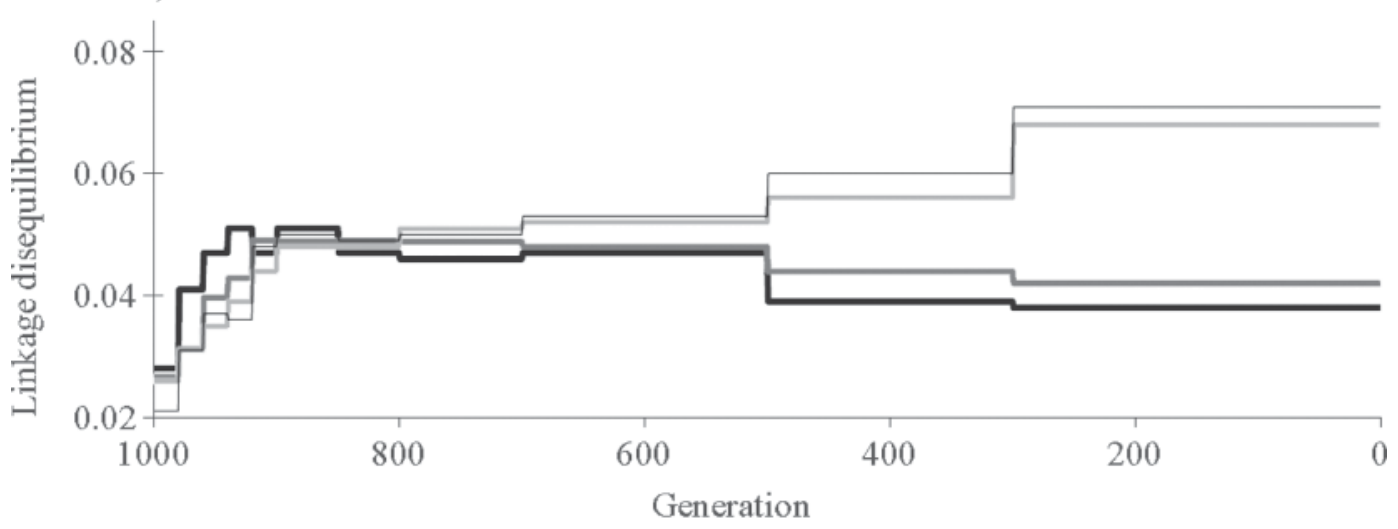

c)

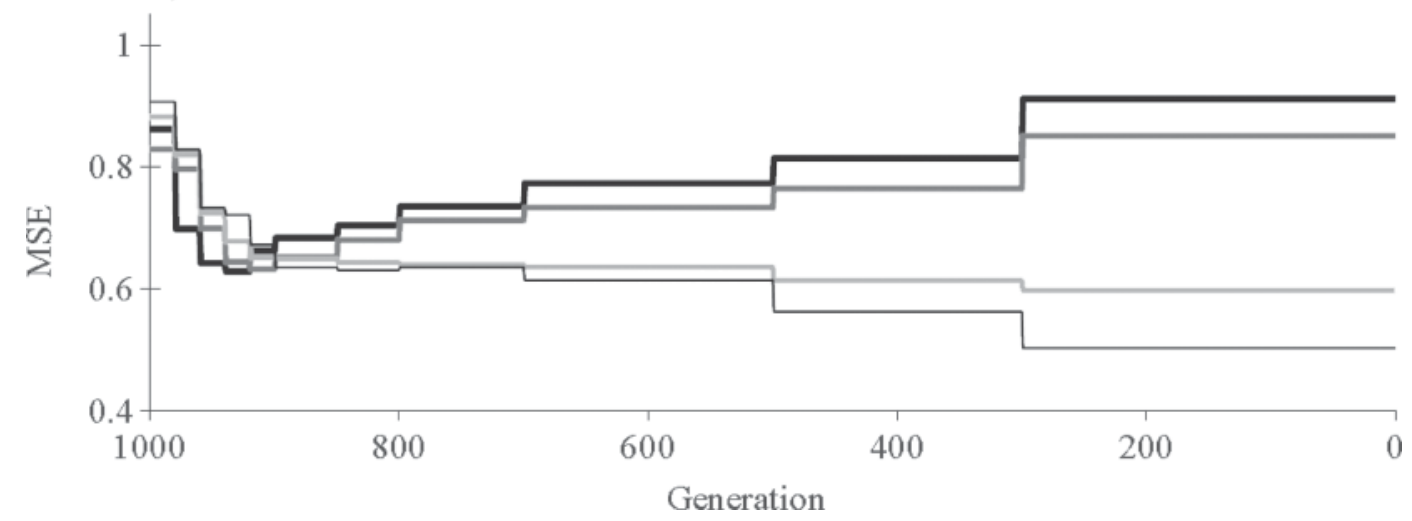

Figure 1. Trends of (a) the mutant QTL allelic frequency, (b) average linkage disequilibrium between QTL and SNP, and (c) standardized mean square error (MSE) by generation when the mutant QTL allele appeared (generation). Simulation scenarios assumed no selection (NSel; thin black line) and positive selection on mutant QTL alleles with low ( $\operatorname{Sel}_{500}$; light gray line), intermediate ( $\mathrm{Sel}_{700}$; dark gray line), and high (Sel$l_{900}$; thick black line) selection intensities.

and Robertson, 1968), plots for LD and allelic frequencies showed remarkable departures (Figures 1a and 1b). When a new mutation occurs, LD with neighbor SNP markers can accumulate generation by generation by increasing the frequency of the haplotype including the new mutation (i.e., genetic drift or positive selection), although excessive time intervals would impair linkage disequilibrium due to recombination events and mutations in SNP markers. Positive selection for allele $q$ (e.g., scenario $S e l_{900}$ ) led to a faster increase of $\mathrm{r}^{2}$ when compared with scenario NSel or scenarios with less intense selection (e.g., $S e l_{500}$ and $S e l_{700}$ ). It is important 
to differentiate the pattern shown by higher and lower selection intensities. Whereas $\mathrm{r}^{2}$ reached its maximum estimates with approximately 90 - and 70-generationold mutations in scenarios $S e l_{700}$ and $S e l_{900}$, respectively, and showed a decreasing trend later, $\mathrm{NSel}$ and $\mathrm{Sel}_{500}$ led to an increase of $r^{2}$ with time; the fastest increase was for new mutations originating during the last 100 generations. Although stronger selection intensities could take advantage of an accelerated accumulation of the mutant QTL and linked SNP alleles along a short period (i.e., it minimized the effect of new recombinations and mutations in the SNP markers), softer or null selection delays these phenomena, increasing the entropic contribution of recombination and new mutations on partially linked loci.

The principal objective of this research relied on the performance of genomic prediction depending on the age of the mutant QTL allele, it being revealed by the MSE statistic. All simulation scenarios provided similar $(P>0.05)$ and poor statistical performance for genomic prediction models for very young mutations (i.e., last 20 generations), although this could be anticipated due to the small linkage disequilibrium estimates shown in Figure 1b. Note that MSE progressively decreased with mutation age under scenario $\mathrm{NSel}$ and reached its maximum performance with the oldest mutations. On the other hand, intense selection in scenario $S e l_{900}$ resulted in a quick decrease of the MSE during the first few generations (up to 920-939; Figure 1c), whereas genomic prediction models accounting for older mutations were penalized with increasing MSE estimates. As for the frequency of the mutant QTL allele, scenario $\mathrm{Sel}_{500}$ agreed with scenario $\mathrm{NSel}$, suggesting a less pronounced decline, whereas scenario $\mathrm{Sel}_{700}$ showed a pattern comparable to scenario $S e l_{900}$. It is important to note that minimum MSE estimates were significantly smaller in scenario NSel $(0.502 \pm 0.020$; generations 1 to 300$)$ than in scenario $\operatorname{Sel}_{900}(0.628 \pm 0.005$; generations 920 to 939 ), whereas overall estimates penalized scenario NSel $(0.722 \pm 0.003$ vs. $0.703 \pm 0.002 ; P<$ 0.05). Both $\mathrm{Sel}_{500}$ and $\mathrm{Sel}_{700}$ showed intermediate performances. Although inference on some specific mutations could be optimized in nonselected populations, overall performance of genomic prediction methodologies must be slightly better when applied on phenotypic traits previously exposed to genetic selection programs or natural selection. Maximum response to genomic selection must be obtained in traditionally selected traits (e.g., milk-related traits; VanRaden et al., 2009) or fitness-related traits (e.g., reproduction traits such as daughter pregnancy; VanRaden et al., 2009), whereas the application of genomic selection to traits that are hard to select for under traditional BLUP method- ologies (e.g., meat quality in pigs; Davoli and Braglia, 2007) must deal with a penalization linked to the lack of previous selection. This does not invalidate the usefulness of genomic selection for such nonselected traits but suggests a lower response to selection compared with traditionally selected traits.

The change of MSE reported in Figure 1c suggested highly relevant characteristics of genomic prediction with remarkable consequences on current breeding programs: (1) a reduced ability to capture additive genetic variability from young mutations and even from old mutations under moderate-to-strong selection, and (2) differential behavior of genomic prediction models determine if the phenotypic trait was previously exposed to genetic selection (i.e., null or low selection intensity). Accounting for genetic variability from recent mutations in mixed linear models suffered from important limitations due to the restricted amount of linkage disequilibrium between SNP markers and the QTL (Figure 1b) as well as more extreme allelic frequencies (Figure 1a). These limitations impaired the statistical performance of genomic prediction and led to a $77.1 \%$ (scenario $N S e l$ ) or $37.3 \%$ increase (scenario $S e l_{900}$ ) of MSE compared with the corresponding minimum MSE shown in Figure 1c. These were important drawbacks when considering the substantial and continuous uploading of new mutations contributing additive genetic variability in both experimental (Casellas and Medrano, 2008) and livestock species (Casellas et al., 2010). Note that a relevant amount of the genetic variance for phenotypic traits under genomic selection would arise during the last few generation (see the distribution of mutation ages in Table 1), implying a substantial source of partially unaccounted-for genetic effects by genomic prediction models. Standard BLUP models suffer from the same limitation; that is, they traditionally derive genetic inference from the genetic variability of the founder population (Henderson, 1973), although recent parameterization (Wray, 1990; Casellas and Medrano, 2008) may allow for a proper inference on subsequent mutations and improve the performance of genetic evaluation (Casellas et al., 2010). Further efforts to accommodate these recent and unaccounted-for mutations in genomic prediction models would be of special interest for genetic improvement programs to provide a better prediction of the breeding values and response to selection.

The differential behavior of MSE between scenarios NSel, Sel $l_{500}, \mathrm{Sel}_{700}$, and $\mathrm{Sel}_{900}$ must be of special interest depending on the nature of the phenotypic traits under genomic selection. Whereas the development of new parameterizations to account for recent mutations is essential for previously unselected or poorly selected 
traits, they would be less relevant for traits having experienced effective selection (Figure 1c).

\section{ACKNOWLEDGMENTS}

The research contract of J. Casellas was partially financed by Spain's Ministerio de Ciencia e Innovación (program Ramón y Cajal, reference RYC-2009-04049). This research was funded by grants AGL2010-15903/ GAN and AGL2010-21176/GAN (Ministerio de Ciencia e Innovación, Spain). The English revision by C. Ng is also acknowledged.

\section{REFERENCES}

Adams, J. C., W. S. Brainerd, J. T. Martin, B. T. Smith, and J. L. Wagener. 1992. Fortran 90 Handbook. Intertext Publications, New York, NY.

Arias, J. A., M. Keehan, P. Fisher, W. Coppieters, and R. Spelman. 2009. A high density linkage map of the bovine genome. BMC Genetics 10:18.

Casellas, J., G. Caja, and J. Piedrafita. 2010. Accounting for additive mutations on litter size in Ripollesa sheep. J. Anim. Sci. $88: 1248-1255$.

Casellas, J., and J. F. Medrano. 2008. Within-generation mutation variance for litter size in inbred mice. Genetics 179:2147-2155.

Davoli, R., and S. Braglia. 2007. Molecular approaches in pig breeding to improve meat quality. Brief Funct. Genomic Proteomic $6: 313-321$.

Falconer, D. S., and T. F. C. Mackay. 1996. Introduction to Quantitative Genetics. Longmans Green, Harlow, Essex, UK.

Gelfand, A., and A. F. M. Smith. 1990. Sampling based approaches to calculating marginal densities. J. Am. Stat. Assoc. 85:398-409.

Gianola, D., M. Pérez-Enciso, and M. A. Toro. 2003. On markerassisted prediction of genetic value: beyond the ridge. Genetics 163:347-365.

Habier, D., R. L. Fernando, and J. C. M. Dekkers. 2009. Genomic selection using low-density marker panels. Genetics 182:343-353.

Hayes, B. J., and M. E. Goddard. 2001. The distribution of effects of genes affecting quantitative traits in livestock. Genet. Sel. Evol. 33:209-229.
Henderson, C. R. 1973. Sire evaluation and genetic trends. Pages 10 41 in Proc. Animal Breeding and Genetics Symposium in Honor of Dr. Jay L. Lush. ADSA-ASAS, Champaign, IL.

Henderson, C. R. 1976. A simple method for computing the inverse of a numerator relationship matrix used in the prediction of breeding values. Biometrics 32:69-83.

Hill, W. G., and A. Robertson. 1968. Linkage disequilibrium in finite populations. Theor. Appl. Genet. 38:226-231.

Ibáñez-Escriche, N., R. L. Fernando, A. Toosi, and J. C. M. Dekkers. 2009. Genomic selection of purebreds for crossbred performance. Genet. Sel. Evol. 41:12.

Innan, H., and M. Nordborg. 2003. The extent of linkage disequilibrium and haplotype sharing around a polymorphic site. Genetics 165:437-444.

Kass, R. E., B. P. Carlin, A. Gelman, and R. Neal. 1998. Markov chain Monte Carlo in practice: A roundtable discussion. Am. Stat. $52: 93-100$

Kosambi, D. 1944. The estimation of map distances from recombination values. Ann. Eugen. 12:172-175.

Meuwissen, T. H. E., B. J. Hayes, and M. E. Goddard. 2001. Prediction of total genetic value using genome-wide dense marker maps. Genetics 157:1819-1829.

Ødegård, J., A. K. Sonesson, M. H. Yazdi, and T. H. E. Meuwissen. 2009. Introgression of a major QTL from an inferior into a superior population using genomic selection. Genet. Sel. Evol. 41:38.

Quaas, R. L. 1976. Computing the diagonal elements and inverse of a large numerator relationship matrix. Biometrics 32:949-953.

Raftery, A. E., and S. M. Lewis. 1992. How many iterations in the Gibbs sampler? Page 763 in Bayesian Statistics IV. J. M. Bernardo, J. O. Berger, A. P. Dawid, and A. F. M. Smith, ed. Oxford University Press, Oxford, UK.

Sargolzaei, M., F. S. Schenkel, G. B. Jansen, and L. R. Schaeffer. 2008. Extent of linkage disequilibrium in Holstein cattle in North America. J. Dairy Sci. 91:2106-2117.

Toosi, A., R. L. Fernando, and J. C. M. Dekkers. 2010. Genomic selection in admixed and crossbred populations. J. Anim. Sci $88: 32-46$

VanRaden, P. M., C. P. Van Tassell, G. R. Wiggans, T. S. Sonstegard, R. D. Schnabel, J. F. Taylor, and F. S. Schenkel. 2009. Invited review: Reliability of genomic predictions for North American Holstein bulls. J. Dairy Sci. 92:16-24.

Wray, N. R. 1990. Accounting for mutation effects in the additive genetic variance-covariance matrix and its inverse. Biometrics $46: 177-186$. 